Horizons philosophiques

\title{
Autour de Socrate(s). Rencontre avec Sarah Kofman
}

\section{Ghyslaine Guertin}

Volume 10, numéro 2, printemps 2000

Le savoir en fête

URI : https://id.erudit.org/iderudit/802934ar

DOI : https://doi.org/10.7202/802934ar

Aller au sommaire du numéro

\section{Éditeur(s)}

Collège Édouard-Montpetit

\section{ISSN}

1181-9227 (imprimé)

1920-2954 (numérique)

Découvrir la revue

\section{Citer ce document}

Guertin, G. (2000). Autour de Socrate(s). Rencontre avec Sarah Kofman. Horizons philosophiques, 10(2), 53-64. https://doi.org/10.7202/802934ar d'utilisation que vous pouvez consulter en ligne.

https://apropos.erudit.org/fr/usagers/politique-dutilisation/ 


\section{AUTOUR DE SOCRATE(S) RENCONTRE AVEC SARAH KOFMAN}

Sarah Kofman a bien voulu accorder à La petite revue de philosophie cet entretien 1 autour de son tout dernier ouvrage intitulé Socrate(s)2 ${ }^{2}$, Sur le ton de la conversation, elle nous fait découvrir son interprétation de Socrate à partir des Socrate(s). Le lecteur comprendra pourquoi nous avons tenu à publier ses propos qui viennent à la fois clore et ouvrir à nouveau le débat sur la connaissance de soi. L'interprétation philosophique serait-elle inconciliable avec celle qu'en fait la psychologie ou la psychanalyse? Un retour aux sources s'impose. Sarah Kofman les a retracées en démontrant la pluralité des intrigues et des significations autour du Philosophe.

G.G. : Sarah Kofman, j'aimerais au départ que vous nous situiez cet ouvrage par rapport à l'ensemble de votre production et de votre cheminement intellectuel. Nous connaissons vos préoccupations pour l'art, le féminisme, la psychanalyse de même que votre intérêt pour des philosophes comme Nietzsche, Auguste Comte, pourquoi alors Socrate?

S.K. : Mon intérêt pour Socrate apparaît complètement nouveau par rapport à tout ce que j'ai fait jusqu'à présent. Pourtant, en réalité, ce livre n'est pas vraiment uniquement sur Socrate, mais sur les différentes lectures de Socrate au cours de l'histoire faite par les philosophes. C'est autant un livre sur Hegel, Nietzsche et Kierkegaard que sur Socrate et Platon. C'est Socrate réinterprété. Et ce qui m'intéresse dans Socrate, c'est surtout la réappropriation qu'en a fait la philosophie au cours du temps. Cela n'est pas très nouveau chez moi, ce rapport de lecture, puisque dès le début, le

1. Réalisé à Paris le 17 mars 1989.

2. Paris : Éditions Galilée, 1989, 337 p. 
premier texte que j'ai publié, c'était, d'abord sous forme d'article, «Freud et Empédocle», qui mettait déjà en rapport le philosophe antique, Empédocle, dit pré-socratique, et la psychanalyse. Ensuite, dans mes différentes lectures des textes de Freud, par exemple dans la littérature, j'ai toujours fait une relecture des lectures de Freud. J'ai d'ailleurs intitulé ce livre - qui s'appelle Quatre romans analytiques justement pour montrer comment Freud au lieu d'expliquer notamment les sources, se les appropriait pour en faire de véritables romans. En fait, je peux dire que si maintenant après mon dix-neuvième livre, je devais me définir (ce que je n'aime pas faire [...]) je pourrais dire que ma méthode est une méthode herméneutique au second degré (...).

Très dernièrement, j'ai écrit un texte que vous ne connaissez pas peut-être encore (...) qui s'appelle «Nietzsche et l'obscurité d'Héraclite", qui a paru dans une revue suisse, Furor no 15 , et dans lequel je fais exactement ce type de travail à propos de l'obscurité d'Héraclite. Je montre comment toute la tradition philosophique - Platon, Aristote, Hegel toujours, Nietzsche aussi et Heidegger - s'était réapproprié cette notion d'obscurité d'Héraclite. Chacun l'interprétait en rapport avec son propre système. Au fond, de plus en plus, ce qui m'intéresse, c'est ce double intérêt permanent et pour l'Antiquité - Platon, Socrate, les présocratiques - et la modernité. C'est ce qui explique que j'ai été amenée à faire ce travail-là et avec cette méthode d'interprétation au second degré. Pourquoi au second degré? Parce qu'il y a déjà, par exemple, pour Socrate, les différents auteurs qui eux ont déjà chacun lu Socrate à leur manière. Socrate qui n'a pas écrit lui-même, a donc été lu à travers les textes d'autres. À travers Platon, Xénophon ou Aristophane, qui en ont déjà fait une interprétation. Après, il y a mon interprétation de leur interprétation. Ce qui fait qu'à mon tour, j'ai mon Socrate, mes Socrate(s). C'est pour cela que j'appelle cette méthode, une interprétation au second degré. Tout cela pour montrer que j'ai en fait une très grande continuité dans mon travail. 
Deuxièmement, par rapport à mon autre centre d'intérêt, les femmes, ce n'est pas du tout exclu de ce livre-là, puisqu'en réalité, mon type d'interprétation que j'appellerais une interprétation au second degré, c'est une interprétation symptomale. Je veux dire que les différents écrits des différents auteurs que j'envisage dans leur réappropriation de Socrate, je les vois comme des symptômes de ce qu'ils sont eux-mêmes et des symptômes de leurs pulsions, à la fois au sens nietzschéen qu'au sens freudien du terme. Je montre, en particulier pour la figure de Socrate, que finalement si cette figure de Socrate les gêne tous d'une manière ou d'une autre, c'est parce que c'est une position atopique, déjà Platon le disait. (...) Cette position atopique, ou atypique dans le cas de Nietzsche, les gêne dans leur système. Et si cela les gêne, c'est parce que cela remet quelque chose en question dans leur propre équilibre pulsionnel et, plus particulièrement, dans leur relation aux femmes et à la féminité en eux. (...) Je ne suis pas une féministe engagée, ce qui m'intéresse c'est la femme à l'intérieur de la philosophie et le refoulement par les philosophes de la féminité en eux. Et quand ils ne refoulent plus, la transformation que cela entraîne dans leur propre système. Et je montre la même chose à propos de Socrate. À chaque fois, que ce soit pour Nietzsche, pour Kierkegaard ou pour Hegel, c'est cela qui est en jeu. Et alors symptomatiquement de cette chose, c'est par exemple déjà, le sort ou le non-sort donné à Xanthippe, la femme de Socrate. Pratiquement aucun d'eux, sauf Nietzsche qui lui donne comme rôle, un rôle extrêmement important au sens où étant donné que c'était une femme épouvantable, hargneuse et colérique, Socrate la fuyait et il était toujours dehors avec les hommes et c'est comme cela qu'il a été amené à inventer la dialectique. (...) Nietzsche en parle de cette manière-là, mais personne ne met en lumière le fait qu'il était bigame. Lorsqu'on lit des sources, c'est-àdire Xénophon ou Diogène Laërce en particulier, Platon n'en parle que dans le Phédon, pour l'évacuer, et tous les autres font la même chose finalement; même Nietzsche, soi-disant 
l'anti-platonicien, fait pareil. On évacue les femmes et les enfants, ce n'est pas important et même le fait que Socrate soit bigame et qu'il était passionné (...). Ce qui les intéresse tous beaucoup plus, c'est le couple qu'il forme avec Platon et la position plus ou moins féminine qu'il occupe dans ce couple (...). En tout cas, une position oscillante et cela les gêne énormément, chacun dans son système. Tout cela pour vous dire qu'en fait, je ne perds aucun de mes centres d'intérêts habituels.

G.G. : Votre livre, Sarah Kofman, comporte quatre parties : I- Les Socrate(s) de Platon; II- Le Socrate "Bifrons" de Hegel; III- Le Socrate de Kierkegaard; IV-Les Socrate(s) de Nietzsche. Mais n 'accordez-vous pas une place privilégiée à Kierkegaard?

S.K. : (...) Kierkegaard est privilégié en volume parce que lui-même a écrit un très gros livre sur Socrate (Le concept d'ironie3). Nietzsche est un petit peu partout et Platon, je le retrouve à l'intérieur de Kierkegaard. En plus, Kierkegaard répond à Hegel, D'autre part, Kierkegaard est le seul à avoir pris en considération, comme essentielle, l'ironie dans Socrate. L'ironie, c'est-à-dire ce qui les a tous gênés. L'ironie qui est inclassable. Mais cela ne veut pas dire que ma position est celle de Kierkegaard. Si... Parce que, ce que je montre, c'est que tout le monde élimine quelque chose dans Socrate. Lui, quand il insiste sur un Socrate totalement négatif, l'ironie, c'est quand même un point de vue chrétien chez lui. C'est essentiellement pour le bien distinguer du Christ d'un côté et de Platon de l'autre. Donc, il y a quelque chose en lui qui résiste à Socrate. II résiste même à l'ironie (...) parce que l'ironie n'est qu'un moyen, c'est une vertu du commencement et ce n'est pas quelque chose à laquelle on devrait se tenir. Ce serait dépassé par le stade religieux et éthique. En ce sens, il y a une résistance à Socrate. Mais il a vu la place fondamentale de l'ironie quand même. (...) En tant que chrétien, Kierkegaard ne peut supporter, en particulier, dans l'ironie : la duplicité, la non-transparence (...).

3. Publié en français chez l'Oronte sous le titre de Le concept d'ironie constamment rapporté à Socrate. 
G.G. : Revenons, si vous le voulez, aux Socrate(s) de Platon et, plus particulièrement, au problème de l'interprétation du "connais-toi toi-même». Quel sens lui avez-vous accordé? Vous êtes-vous référé, pour votre interprétation, à l'Alcibiade?

S.K. : Je n'ai pas accordé une place fondamentale à l'Alcibiade. J'ai fait le discours d'Alcibiade dans le Banquet. Je me réfère de temps en temps à l'Alcibiade, mais le "connais-toi toi-même" dans l'Apologie de Socrate, essentiellement où il en est question, renvoie à l'autre. Connais-toi toi- même, mais comme miroir. C'est quand même important cette idée, même si je n'en fais pas un très long développement, dans la mesure où ma lecture du Banquet de Platon et l'interprétation que je donne d'Alcibiade montrent, et c'est je crois assez original, que Socrate et Alcibiade sont des doubles l'un de l'autre. En particulier, Socrate représente la part d'Alcibiade qu'il n'a pas acceptée, c'est-à-dire le logos, et qu'il a projetée à l'extérieur de lui sous forme de Socrate, d'un double qui le persécute, qu'il recherche. Et si on se réfère à l'Alcibiade de Platon, on peut voir cela d'assez près, dans la mesure où Socrate dit à Alcibiade qu'on ne peut se connaître qu'à travers l'autre et dans le miroir de l'autre, et que tant qu'Alcibiade le fréquente, il ne perdra pas son âme. Donc en réalité, le miroir c'est le double. Et s'il ne peut se connaître sans Socrate, c'est parce que Socrate est son double en tant que logos, en tant que la partie intellectuelle de lui-même. J'ai montré très en détails comment, dans le Banquet, Alcibiade fuit Socrate parce qu'il se fuit lui-même et il ne veut pas de réconciliation. D'autre part, dans les images qu'il donne de Socrate, le Silène et le Marsyas, ce sont des images inversées de celles que Socrate donne de lui-même dans l'Alcibiade, c'est-à-dire, en particulier, du peuple. II dit dans l'Alcibiade, qu'Alcibiade recherche le peuple qui a des beaux dehors mais dont l'intérieur est pourri. Socrate, lui, a des dehors laids et est beau à l'intérieur. Donc, il renverse l'image que Socrate donne lui-même du "démos" dans 
l'Alcibiade. En ce qui concerne le connais-toi toi-même, je montre comment les différents philosophes l'interprètent chacun à leur manière différente. C'est-à-dire que, à l'intérieur de Socrate et de Platon, on peut dire qu'on retourne à n'oublie pas que tu es une pensée, (l'âme comme pensée), et donc c'est justement ce rapport au miroir et qu'Alcibiade fuit et projette comme un double persécuteur. (...)

Pour Hegel, ce souviens-toi toi-même, c'est l'oracle d'Apollon qui le dit, et il voit ce souviens-toi toi-même comme l'appel au retour de l'intériorité. II interprète déjà l'appel à la réminiscence platonicienne (...) comme le retour à la conscience, à l'intériorité (...), le retour à ce qu'il appelle l'oracle intérieur par opposition à l'oracle extérieur. Mais en même temps, Hegel montre que cet oracle intérieur, cet appel au souvienstoi toi-même est pensé comme oracle et, ce, parce qu'il se réfère à Xénophon comme source (...), et pas à Platon. II en appelle donc à Xénophon et, à ce moment, il comprend le souviens-toi toi-même comme un appel à la conscience. Mais comme c'est le premier moment de la conscience qui n'est pas encore tout à fait conscience - elle commence seulement à s'éveiller -, cette intériorité, il la perçoit d'abord comme une voix intérieure, démoniaque, qui n'est pas perçue comme conscience. Donc comme un oracle intérieur qui s'oppose aux oracles extérieurs (...). II a été condamné pour cela, puisque c'était une autre forme d'oracle. Ensuite, il faut bien que dans sa dialectique, il montre qu'une fois Socrate mort, les Grecs lui ont édifié une statue et puis (...) on est passé à un stade supérieur de conscience. Donc une fois qu'il est mort, le phénix est né de nouveau (...), dans la mesure où en réalité ce qu'il apportait sans le connaître encore lui-même, c'était le principe de l'universel. Mais à ce moment-là, il a recours à Platon et non plus à Xénophon. II y a donc chez Hegel, ce va-et-vient de sources, selon qu'il veut montrer une chose ou une autre (...). Chez Kierkegaard, le connais-toi toi-même c'est le démon (...), le retour à soi est toujours un retour vers soi, comme rien, comme néant - 
c'est ironique (...). Kierkegaard donne la version platonicienne du démon comme quelque chose de négatif, il se tourne vers le rien. Quant à Nietzsche, le démon prend lui aussi la lecture platonicienne (...). Mais c'est aussi un démon inhibiteur, négatif qui arrête sur la voie de l'action. II voit que l'idée du démon chez Socrate apparaît à travers l'ironie de Socrate. Socrate qui prône toujours le retour à la raison, à la dialectique comme unique chemin de la vertu. À un moment donné, il faut prendre cela de façon ironique parce que Socrate savait très bien que la raison n'est pas quelque chose qui peut être un principe d'action et qui ne donne donc pas de fin. C'est un calcul, la raison, mais elle ne peut pas donner des fins. II n'y a que l'instinct qui peut donner des fins. Et quand Socrate en appellerait au démon, ce serait pour montrer les limites de la raison. Et pour lui, le connais-toi toi-même, l'oracle d'Apollon ne veut surtout pas dire, ni connais-toi comme pensée, ni connais-toi comme conscience, ni connais-toi comme universel. Si c'était cela, Nietzsche dit qu'on aurait vite fait le tour de la conscience parce qu'elle est complètement superficielle, il n'y a rien dedans. Mais pour lui cela veut dire connais les limites de la raison, et derrière la raison, il y a les instincts. Seulement, deuxième point, et c'est là que cela ne va plus, il en appelle à l'instinct contre la raison, mais seulement il voit l'instinct de façon négative, inhibitrice, et cela c'est le symptôme de la décadence de Socrate. II est décadent parce que, alors que l'instinct est toujours positif et la conscience critique est dissolvante, il fait l'inverse.

G.G. : Où se logerait alors le «vrai» Socrate? On le devine bien, il n'y en a pas...

S.K. : Il n'y en a pas. À chacun son Socrate toujours en rapport aux textes qui sont soit de Platon, de Xénophon ou d'Aristophane. Kierkegaard montre admirablement comment chacun des trois dites sources sont déjà des Socrate(s) vus à travers un stéréoscope différent, mises en relief différentes (...). Autant Nietzsche et Hegel se servent de Xénophon, autant Kierkegaard en a horreur parce qu'il montre un 
Socrate complètement ennuyeux, boutiquier, calculateur, utilitaire, trop positif dans le sens positiviste du terme. Platon au contraire l'a trop idéalisé - comme l'a montré Nietzsche. Et quant à Aristophane, pour Kierkegaard, c'est la source la meilleure parce qu'elle a bien montré un Socrate complètement négatif. Mais, il montrera bien qu'Aristophane ne l'a pas réduit à la figure d'un sophiste. Car pour Kierkegaard, le sophiste est encore une figure positive puisqu'elle renvoie toujours en dernière analyse à l'utile (...).

G.G. : Nous sommes bien éloignés d'une interprétation "psychologique" du connais-toi toi-même; comment expliqueriez-vous alors la tendance qu'on a aujourd'hui à voir le connais-toi toi-même comme un acte introspectif?

S.K. : Si on rattache le connais-toi toi-même à la psychologie, on transforme l'acte réflexif en introspection (...). D'autre part, déjà réinterprété par Descartes, le connais-toi toi-même, on voit bien que le cogito est un acte de la pensée, ça n'a jamais été un acte d'introspection. Je me demande comment on tombe dans cette aberration. Je crois que l'erreur doit venir du fait qu'il y ait une adresse, une adresse avec un tu - connais-toi toi-même. Or, au niveau de l'universel ce n'est pas un toi, pas un je. Je crois que l'erreur doit venir de là. C'est cet impératif apostrophique, si j'ose dire, qui est devenu catastrophique, parce qu'on a entendu, connais-toi toi-même. C'est un appel à un retour sur soi comme individu. Ce qui est curieux, c'est qu'il y ait un appel à travers un tutoiement. Alors, c'est de l'ordre de l'impératif moral, parce que quand c'est un impératif moral, il y a toujours une adresse directe à un sujet parce que c'est lui qui est responsable d'une action et personne d'autre. Donc, l'impératif kantien, par exemple, l'impératif moral est toujours un impératif qui s'adresse à l'individu : fais ceci... agis de telle sorte. Quand il s'agit d'une action, l'impératif c'est toujours une voix qui s'adresse nécessairement à un individu même si cet individu doit obéir à une loi qui, elle, est universelle et qui est la loi de la raison. Mais le passage de l'universel de la loi, en tant qu'impératif, à la réalisation de 
cet impératif, passe nécessairement par une action individuelle. C'est pourquoi la loi passe par un mobile. II y a le principe, le motif, le mobile chez Kant, et le mobile c'est ce qui permet de passer du principe universel à la maxime. Et le mobile en tant que sentiment - même si c'est un sentiment a priori -, le respect s'adresse à un individu (...). Et je pense que le connais-toi toi-même est donné comme un impératif moral. Et la figure de Socrate, c'est celle du fondateur de la morale. (...) C'est : connais-toi toi-même comme quelqu'un qui est autonome. C'est toi qui vas donner la décision de ton action et tu n'auras plus à obéir à la loi de la cité. C'est en tant que cela passe par un impératif de la morale et comme fondateur de la morale. Même si on le voit comme un oracle intérieur, l'oracle intervenait dans l'action et pas dans la pensée. Ensuite, quand on passe du connais-toi toi-même comme quelqu'un capable d'agir en prenant ses décisions, quand ça passe au niveau de l'universel, on a tendance à le psychologiser parce que cela s'adresse à un individu. C'est là l'erreur. Ce qui est étonnant aussi c'est de rapprocher la maïeutique socratique avec la psychanalyse. Ça n'a aussi rien à voir (...). Dans la maïeutique, ce qui est important c'est l'interrogation, le questionnement qui va éliminer peu à peu les opinions fausses, en tout cas, faire qu'on les expose pour voir si elles sont viables ou non. C'est une exposition à l'extérieur pour légitimer l'enfant qui naît (...). Dans la psychanalyse, ce n'est pas du tout ce qui se passe. Le psychanalyste se tait, ne pose aucune question. C'est l'autre qui parle tout seul. Alors, ça n'a rien à voir avec la dialectique, rien à voir avec la maïeutique (...). II y a des déviations qui se produisent de façon tout à fait étonnante. Et là je crois que le rapprochement passe par le problème du transfert parce que dans les deux cas il y a transfert, et s'il y a transfert dans l'un comme dans l'autre, ça passe par le silence, par l'aveu de non savoir de Socrate. Le maïeuticien est stérile, il le dit qu'il fait accoucher les autres, mais qu'il n'apprend rien. Tout ce qu'il sait c'est qu'il ne sait rien et c'est aux autres de parler. En tant que tel, c'est un personnage 
silencieux. Moins il le sait, moins il déclare savoir, plus les autres lui transfèrent le savoir. C'est le cas d'Acibiade et de tous les autres. La figure de Socrate comme figure de la maîtrise vient de son désaveu de maîtrise. Au niveau de la psychanalyse, c'est le silence de l'analyste qui fait que quand c'est interprété par Lacan - le psychanalyste se présente comme le "supposé savoir» précisément parce qu'il se tait. Alors moins on donne, plus l'autre vous donne (...). La psychanalyse n'a rien à voir avec un accouchement.

G.G. : Sarah Kofman, on ne peut nier aujourd'hui la séduction qu'exerce Socrate au niveau de la réflexion philosophique. II est souvent présenté comme la figure même du philosophe et, à ce titre, il joue un rôle extrêmement important dans l'apprentissage de la philosophie. Votre ouvrage ne pourrait-il pas être utilisé comme instrument pédagogique?

S.K. : Quand on est professeur de philosophie au Secondaire (j'ai quand même enseigné dix ans au Secondaire, à des étudiants qui n'ont jamais fait de philosophie), on connaît surtout Socrate à travers Platon et on voit Socrate comme la figure même du philosophe (...). C'est déjà une figure réappropriée par Platon qui érige Socrate en maître précisément parce qu'il n'est pas un maître. Et quand on fait le travail à fond comme je l'ai fait, on s'aperçoit que loin d'être la figure type du philosophe, il est justement celui qui résiste à toute philosophie, et c'est cela qui est extraordinaire comme découverte. II est celui dont aucun philosophe ne sait quoi faire et qui résiste à tout système, à toute philosophie. Et comme le dit Hegel, Kierkegaard, Socrate n'a pas de philosophie. II n'a rien à affirmer, il n'a pas de système. II ne fait qu'accoucher. C'est donc l'inverse du philosophe. Quant à Hegel - qui est celui qui veut lui donner la plus grande place - il en fait le tournant fondamental de l'histoire, de l'esprit, mais ce n'est pas le tournant de l'apparition du philosophe mais c'est plutôt l'apparition en tant qu'apparition (...). Il est encore pris complètement de l'autre côté, c'est pourquoi il reste immobile. Cette immobilité extatique 
est interprétée comme une maladie, une maladie nécessaire de l'esprit (...). Or, quand on a des élèves en classe terminale, on ne veut pas du tout qu'ils tombent dans l'immobilité extatique ni qu'ils entendent des voix démoniaques. On voudrait les faire passer directement de la non-philosophie à la philosophie. Et ce que montre très bien Hegel, c'est que ce passage n'est pas possible de cette manière (...). On vient à penser en pensant et, si on passe de la non-pensée à la pensée, cela ne va pas. Et ce qui est très étonnant, c'est que cette figure de Socrate n'est pas la figure du philosophe, sauf chez Platon. C'est le Socrate de Platon qui le fait la figure du philosophe (...). II n'a pas du philosophe une figure de quelqu'un qui affirmerait un savoir mais le philosophe comme amour, comme amour du savoir et donc comme recherche. Le philosophe comme recherche, comme pur questionneur et pur penseur. Et c'est comme cela qu'il faut le voir. Si on a une conception de la philosophie comme Hegel comme savoir absolu, alors ce n'est surtout pas cela. C'est la figure du questionnement indéfini (...). Ce qui est intéressant à montrer aux élèves c'est que pour toute conception de la philosophie comme système, comme savoir, il n'est pas récupérable et quand on veut le récupérer, on en fait quelque chose d'autre. (...) Le problème est de savoir si nous pouvons avoir encore aujourd'hui la même conception du philosophe que celle de Platon. Parce qu'en fait, c'est le Socrate de Platon qu'on a là. Est-ce qu'on peut avoir comme philosophie, une philosophie interrogative, une recherche qui n'aboutit à rien? Mais si on en croit Kierkegaard, et je suis très près de Kierkegaard là-dessus, c'est de faire une grande différence entre Socrate et Platon; un Socrate purement interrogatif et aporitique, et un Platon qui essaierait de donner un certain savoir, ne serait-ce que sous forme mythique.

Cette entrevue s'est terminée sur une note d'enthousiasme. En faisant référence au travail et à la recherche qui resterait à entreprendre autour de la représentation du Socrate dans l'art. Plus particulièrement dans la peinture et 
la musique. N'y aurait-il pas lieu par exemple, comme l'a montré Jankélévitch, de rapprocher l'ironie de Socrate et celle d'Erik Satie? II s'agirait d'un excellent prétexte pour réunir la philosophie et la musique.

\section{Ghyslaine Guertin}

Professeure au département de philosophie

Collège Édouard-Montpetit 